\title{
Universal Biochip Readout of Directed Hamiltonian Path Problems
}

\author{
David Harlan Wood ${ }^{1 \star}$, Catherine L. Taylor Clelland ${ }^{2}$, and Carter Bancroft ${ }^{2}$ \\ 1 Computer and Information Science \\ University of Delaware \\ Newark, DE 19716 USA \\ woodecis.udel.edu \\ 2 Department of Physiology and Biophysics, Box 1218. \\ Mount Sinai School of Medicine. \\ One Gustave L. Levy Place, New York, NY 10029 USA \\ \{Catherine.Clelland, Carter.Bancroft\}omssm. edu
}

\begin{abstract}
A universal design for a biochip that reads out DNA encoded graphs is enhanced by a readout technique that may resolve multiple solutions of Hamiltonian path problems. A single laboratory step is used. DNA encoded graphs are labeled with many quantum dot barcodes and then hybridized to the universal biochip. Optical readouts, one for each barcode, yield multiple partial readouts that may isolate individual paths. Computer heuristics then seek additional individual paths.
\end{abstract}

\section{Introduction}

The design of a universal biochip for readout of any DNA encoded graph with $n$ or fewer nodes is presented. We emphasize reading out multiple solutions of Directed Hamiltonian path (DHP) problems. We do not solve DHP problems. We merely wish to read out DHP solutions. However, an innovative simultaneous quantum dot barcode labeling of DHP solutions with $n^{2}$ probes yields partial readouts of the biochip. These multiple partial readouts may fully determine some paths, even when multiple paths are present. Further processing by conventional computers seeks additional paths.

Since the pioneering molecular computation of a DHP problem [1], careful design of execution conditions and DNA encodings has been employed to successfully solve DHP problems using DNA $[2,3,4]$. Detection, but not readout, of solutions of the DHP problem was originally performed by a series of DNA strand purifications followed by gel electrophoresis [1]. However, readout of DHP solutions has received less attention.

DHP readout by biochip hybridization has been suggested [2,5]. However, DHP and other graph problems can have multiple solutions, and this presents an important difficulty in that following hybridization to the biochip, all paths

\footnotetext{
* The following partial support is gratefully acknowledged: NSF Grant No. 0130385, NSF Grant No. 9980092, and DARPA/NSF Grant No. 9725021
} 
become superimposed upon one another, leaving any one path indistinguishable from other paths.

Proposed modifications of the original biochip approach have, in principle, overcome the superposition difficulties. One modification uses biochip readout to direct further laboratory steps, including additional biochip readouts, to eventually isolate all of the individual Hamiltonian paths [5]. This method may require $O(n k)$ laboratory steps to find and read out $k$ paths, each of length $n$.

We have designed a readout procedure using innovative multiple labeling and a universal biochip. We describe here a laboratory approach for readout of DHP problems having multiple solutions. We show resolution of multiple DHP solutions may be obtained from $n^{2}$ partial readouts, one for each of our multiple labels. Heuristics for computational determination of further multiple DHP solutions from our partial readouts are presented. These and further heuristics are under development $[6]$.

\section{Graphs and Their Adjacency Matrix Representations}

A graph is a set of nodes, usually drawn as points, plus a set of edges, usually drawn as arrows joining pairs of nodes. The graph representation we use is the adjacency matrix of a graph. It should be noted that any graph with at most $n$ nodes can be represented by an $n \times n$ adjacency matrix. This is one reason our universal biochip design is able to read out unrestricted graphs (up to size $n$ ) encoded in DNA.

\subsection{The Adjacency Matrix of a Graph}

If there are $n$ vertices in a graph, then we form an $n \times n$ adjacency matrix of 0 s and $1 \mathrm{~s}$. If there is a 1 in the $i$ th row at the $j$ th column, this means that the graph has a directed edge from the $i$ th vertex to the $j$ th vertex. A 0 in that position means there is no such edge.

An example of an adjacency matrix representing a map of airline routes involving four cities is

\begin{tabular}{|c|c|c|c|c|}
\hline & Arrive $c_{1}$ & Arrive $c_{2}$ & Arrive $c_{3}$ & Arrive $c_{4}$ \\
\hline Depart $\boldsymbol{c}_{1}$ & 0 & 1 & 1 & 0 \\
\hline Depart $c_{2}$ & 1 & 0 & 1 & 1 \\
\hline Depart $c_{3}$ & 1 & 0 & 0 & 1 \\
\hline Depart $c_{4}$ & 1 & 1 & 1 & 0 \\
\hline
\end{tabular}

From the city in row 1 , one must go to either city 2 or city 3 . From city 2, one can return to city 1 , or go on to either city 3 or city 4 . From city 3, one must go to city 1 or city 4, where one can either return or continue to city 2 or city 1. 


\subsection{Superpositions of Adjacency Matrices}

Sometimes we are given the superposition of the adjacency matrices of a collection of graphs. Technically, superposition is just the operation of elementwise logical OR applied to two or more adjacency matrices. (Logical OR is defined by 0 OR $0=0,1$ OR $0=0$ OR $1=1$, and 1 OR $1=1$.) That is, we obtain a superposition matrix with a nonzero element in position $i, j$ if and only if at least one of the graphs in the collection has a nonzero element in position $i, j$ of its adjacency matrix.

Note that the airline map in (1) might represent a superposition of three maps: one map for an airline with round robin service, and two more maps for associate airlines providing local shuttle services:

$$
\left(\begin{array}{llll}
0 & 1 & 0 & 0 \\
0 & 0 & 1 & 0 \\
0 & 0 & 0 & 1 \\
1 & 0 & 0 & 0
\end{array}\right), \quad\left(\begin{array}{llll}
0 & 0 & 1 & 0 \\
0 & 0 & 0 & 1 \\
1 & 0 & 0 & 0 \\
0 & 1 & 0 & 0
\end{array}\right),\left(\begin{array}{llll}
0 & 1 & 0 & 0 \\
1 & 0 & 0 & 0 \\
0 & 0 & 0 & 1 \\
0 & 0 & 1 & 0
\end{array}\right) .
$$

But notice that several other collections of adjacency matrices could also be superimposed to produce (1).

It is clear that when multiple adjacency matrices are superimposed there is no general method for recovering the individual adjacency matrices from the superposition. However, in this paper we present a technique for the optical readout of an $n \times n$ universal biochip to be decomposed into $n^{2}$ partial readouts that may decompose superpositions of adjacency matrices describing collections of directed Hamiltonian paths. Individual adjacency matrices from the collections may be isolated in this way.

\section{Universal Graph Biochip and Q-dot Barcode Labels}

Let $n$ be the maximum number of nodes in any graph in a collection of graphs. Suppose each input graph in the collection is encoded in a DNA strand containing encodings of its edges (each edge twice, for convenience). Suppose each edge is encoded by appending the encoding of its departure node to the encoding of its arrival node.

\subsection{Standardized DNA Encoding of Graph Nodes}

DNA sequences encoding each of the $n$ nodes are to be agreed upon in advance, and these DNA sequences are designated $c_{1}, c_{2}, c_{3}, \ldots, c_{n}$. This establishes standard DNA sequences allowing any graph with $n$ or fewer nodes to be encoded in DNA. These standardized sequences only need to be designed once. This means that our universal DNA biochip could be fabricated in quantity without advance knowledge of what graphs it may be used to read out. Only two assumptions are made about the DNA encodings of graphs to be read out. 
1. Specific standard sequences are used to encode the graph edges.

2. Any additional DNA sequences must not hybridize to the standard sequences or their complements.

Thus, we are able to design our own DNA sequences to make them convenient for biochip readout for all graphs.

\subsection{Standardized DNA Encoding of Graph Edges}

DNA Encodings of Biochip Spots. A universal biochip for readout of all graphs (up to size $n$ ) uses standard sequences that need to be designed only once. Each of the $n^{2}$ spots on our biochip contains the DNA sequence complementary to the sequence resulting from appending the sequence encoding some node $c_{i}$ followed immediately by the sequence encoding some node $c_{j}$. This is done for each pair $i$ and $j$, with both $i$ and $j$ running between 1 and $n$. Thus the DNA in the spot in $i$ th row and the $j$ th column on the biochip will preferentially hybridize to input strands that contain the sequence $c_{i}$ immediately followed by the sequence $\boldsymbol{c}_{j}$. Figure 1 below shows the layout of the universal chip in the case of $n=4$.

Arrive $c_{1}$
Depart $c_{1}$
Depart $c_{2}$
Depart $c_{3}$
Depart $c_{4}$$\left(\begin{array}{cccc}c_{1} c_{1} & c_{1} c_{2} & c_{1} c_{3} & c_{1} c_{4} \\ c_{2} c_{1} & c_{2} c_{2} & c_{2} c_{3} & c_{2} c_{4} \\ c_{3} c_{1} & c_{3} c_{2} & c_{3} c_{3} & c_{3} c_{4} \\ c_{4} c_{1} & c_{4} c_{2} & c_{4} c_{3} & c_{4} c_{4}\end{array}\right)$

Fig. 1. Given four standard node sequences, $c_{1}, c_{2}, c_{3}$, and $c_{2}$, the $4 \times 4$ universal biochip would have sequences complementary to those shown.

The appended sequences for the $n^{2}$ spots can be designed and tested for noncrosshybridization without regard for the particular graphs to be read out. One of many methods for obtaining non-crosshybridizing DNA sequences is found in [7].

Non-crosshybridization of DNA sequences for other biochips has been specifically investigated before $[8,9]$. However, our universal design makes stronger demands than prior investigations. Since we are limited to choosing only $n$ node sequences, we are not able to use $n^{2}$ independently chosen DNA edge sequences. Preventing cross-hybridization is more challenging in our case because each of our node sequences is used as one-half of many edge sequences (see Fig. 1).

Encoding Graphs in DNA Strands. A graph is constructed as a DNA strand that includes encodings of all of the edges of the graph. Although it is not strictly necessary, let us assume each edge occurs twice in the graph encoding. Labels (e.g. fluorescent) can be attached to the DNA strand, and the strand hybridized 
to a universal biochip. Each spot on the biochip is complementary to one of the $n^{2}$ possible edges. The spots on the universal biochip are prearranged so that when the graph strand is hybridized to the biochip and the labels are observed, the adjacency matrix of the graph is displayed. An example is shown in Fig. 2.

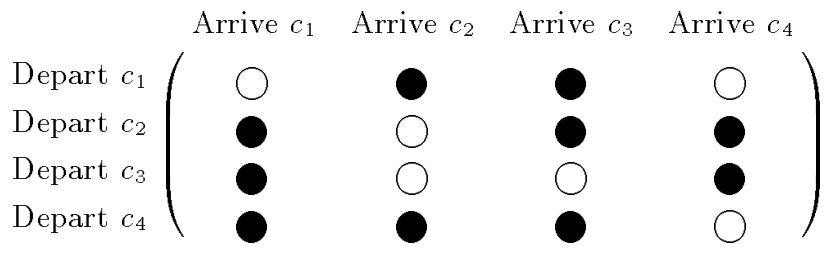

Fig. 2. Appearance of biochip readout for the graph in (1).

\subsection{Fabrication of the Universal Biochip}

Construction of numerous copies of the universal biochip will involve printing each DNA edge sequence onto glass slides using current protocols. Printing 10,000 spots per biochip is possible [10]. Specifically, our DNA sequences to be printed on the biochip can be commercially synthesized, and then printed onto the array in specific positions, using commercial microarray printers, such as the Affymetrix 477 arrayer. (However, the design of the $n^{2}$ complementary edge sequences permits constructing them by ligating $n$ synthesized sequences in pairs.) The use of standard hybridization conditions [8] will allow DNA strands to hybridize to spots on the array that contain sequences complementary to edges encoded within each graph.

\subsection{Quantum Dot Barcodes for Optical Readout}

A fluorescent quantum dot (Q-dot) consists of a nanocrystal cadmium selanide core, wrapped in a zinc sulphur shell [11]. When hit by a beam of light, the electrons in a Q-dot emit light at a specific wavelength (through quantum confinement). The wavelength is directly related to the size of the $\mathrm{Q}$-dot. By varying the shell size, Q-dots with unique, spectrally distinct wavelengths can be produced. A single wavelength of light can be used for simultaneous excitation of all Q-dots [11]. Because Q-dots emit at precise wavelengths, Q-dot emission detection is free of the problems arising from signal overlap or photobleaching [11].

For our purposes, it is important that Q-dots can be integrated into microbeads to form Q-dot barcodes [12] attached to DNA strands. Specifically, Q-dots emitting distinct wavelengths are mixed in known concentrations and incorporated into polystyrene microbeads. This yields beads emitting correspondingly different spectra following excitation. The combinatorial power obtained by varying both the wavelengths and intensities within the fluorescent emission 
spectra of these microbeads yields large numbers of distinct Q-dot barcodes. It has been suggested that "a realistic scheme" could use 5-6 single frequencies, each with 6 intensity levels, yielding approximately 10,000 to 40,000 distinguishable barcodes [12].

For our applications, the Q-dot barcode microbeads can be attached to DNA strands. The carboxylic acid groups on the surface of Q-dot beads can be conjugated to streptavidin molecules, which can then be conjugated to biotinylated DNA strands.

As described in the following section, the optical readout of multiple graphs can exploit the diversity of Q-dot beads that are bound to DNA strands, together forming Q-dot barcode labels.

It is important to note that individual Q-dot barcode microbead images are much smaller than the spots on a typical biochip.

\section{Graph Readout Using the Universal Biochip}

In this section we consider three labeling techniques applicable to collections of graphs each of which is encoded in single stranded DNA. In Subsection 4.1 we use simple uniform labeling of graphs. In this case, all graphs in the collection are superimposed on the biochip. This can give good information if there are very few distinct graphs. In Subsection 4.2 we label only the graphs that contain a specific edge. In this case, only the graphs containing the specific edge are superimposed. This is most useful if only one, or a few, graphs contain the specific edge. The total information is limited, however, by the choice of the specific edge. In Subsection 4.3, we simultaneously label all possible edges in all of the graphs. Again, all graphs in the collection are superimposed, but if each edge label is distinct, we can isolate separate readouts (corresponding to Subsection 4.2) for each possible edge.

The visually oriented reader may want to glance ahead to the examples shown in Fig. 4 and Fig. 5.

\subsection{Labeling all Graphs in a Collection}

Particularly if we have only one DNA encoded graph we wish to read out, it suffices to simply label all the input DNA strands. If there is more than one graph in the input the effect is that all the graphs will be superimposed on the biochip. If there are very few distinct graphs, this may be adequate.

\subsection{Labeling a Single Specific Edge}

Let us concentrate on the labeling of one specific edge. Focus on only those graphs encoded in DNA strands which contain the encoding of the edge $c_{i} \rightarrow c_{j}$. Now let us add a label consisting of DNA complementary to the encoding of the edge $c_{i} \rightarrow c_{j}$. This label is attached to a unique Q-dot barcode microbead. This barcode label can hybridize to any input strand that encodes the edge $c_{i} \rightarrow c_{j}$. 

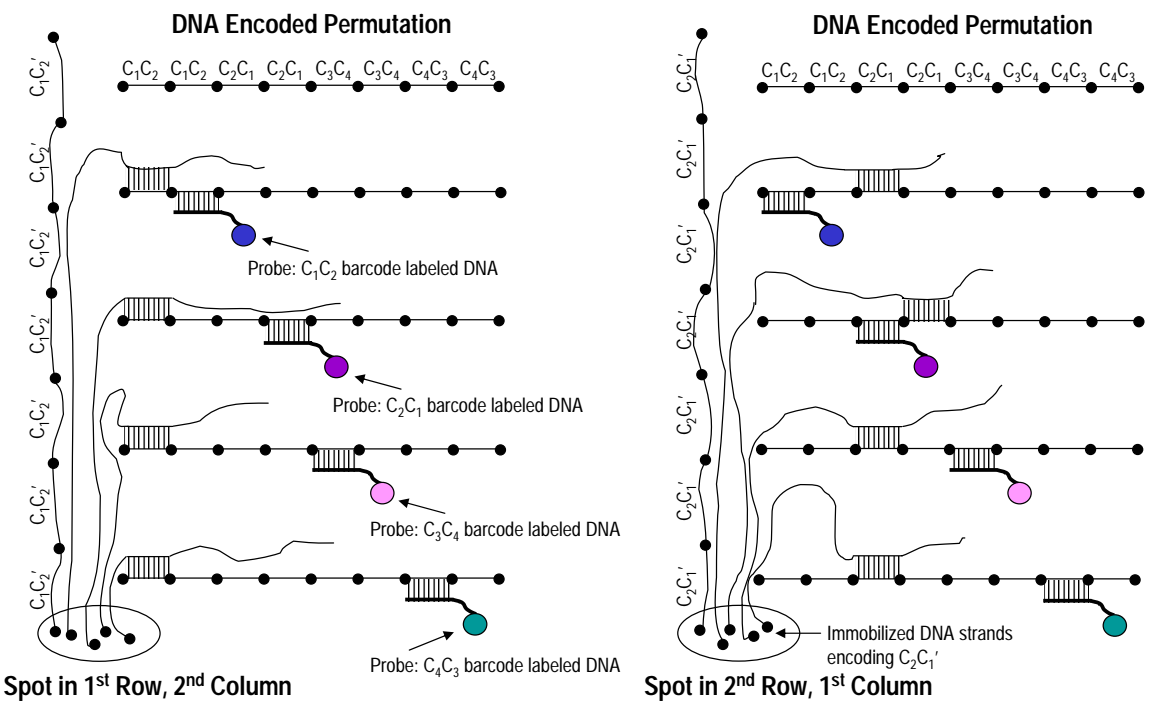

Fig. 3. A labeled graph hybridized to two typical spots on the universal biochip.

All it takes for a spot at the $k, l$ position on the chip to light up is that some graph in the input mixture somewhere contains $c_{i}$ followed by $c_{j}$ (to bind to the $c_{i} \rightarrow c_{j}$ barcode label) and somewhere contains $c_{k}$ followed by $c_{l}$ (to bind to the $k, l$ spot). Recall we are using just this one label, namely, on the DNA encoding the edge from $c_{i} \rightarrow c_{j}$. When we view the whole chip, we see the superposition of all the input graphs that somewhere contain $c_{i}$ followed by $c_{j}$.

The overall effect is that a label corresponding to a particular edge produces the superposition of all graphs containing that edge.

\subsection{Labeling All Possible Edges Simultaneously}

In the previous subsection a single specific edge was barcode labeled. But in this subsection all edges are labeled. We present a technique where $n^{2}$ different Q-dot barcode labels are used, one label for each of the possible edges that can occur in a graph. We apply all of these labels simultaneously in one laboratory step. The labels are scarce enough that the DNA encoded graph strands bind to only one, or a few, labels. Labeling is followed by hybridization to the universal biochip. This sets the stage for Section 5, where a single optical readout is separated into $n^{2}$ images of the biochip, one image for each different barcode edge label.

Figure 3 shows how our scheme using multiple labels interconnects with hybridization to the universal biochip. Two examples are shown in Fig. 3, namely, the biochip spots immobilizing DNA complementary to DNA encoding (a) the edge from $c_{1} \rightarrow c_{2}$ and (b) the edge from $c_{2} \rightarrow c_{1}$. At the top of Fig. 3, as in all the horizontal lines, we have the DNA encoding of a particular permutation graph. 
(Notice that each edge of the permutation graph is encoded twice.) Watson-Crick complements are indicated by primes. For example, $C_{1} C_{2}^{\prime}$ is the complement of $\mathrm{C}_{1} \mathrm{C}_{2}$. Each of the edges $\mathrm{C}_{1} \rightarrow \mathrm{C}_{2}, \mathrm{C}_{2} \rightarrow \mathrm{C}_{1}, \mathrm{C}_{3} \rightarrow \mathrm{C}_{4}$, and $\mathrm{C}_{4} \rightarrow \mathrm{C}_{3}$, is assigned its own unique $\mathrm{Q}$-dot barcode. Recalll the barcoded labels are added so that most graph strands have only one label bound. The left of the figure shows the encoded graph, with various barcode labels, hybridizing to the spot in the first row and second column of the biochip. The right hand side of the figure corresponds to the spot in second row and first column of the biochip.

From Fig. 3 it is seen that when encoded as a DNA strand, a graph literally carries a barcode label corresponding to a particular edge to every spot on the biochip that corresponds to some edge of that graph. Or, in the words of one of the reviewers of this manuscript, "each strand is multiply indexed: once by its position on the chip (which identifies one edge in the encoded Hamiltonian path $(i, j)$ ), and a second time by an attached label (which identifies a second edge in the encoded Hamiltonian path $(k, l))^{\text {" }}$

\subsection{Example Readouts Using Universal Biochip}

Figure 4 schematically shows examples of biochip readouts using $n^{2}$ different Qdot edge barcode labels (Q-dots enlarged about one thousand-fold for clarity).

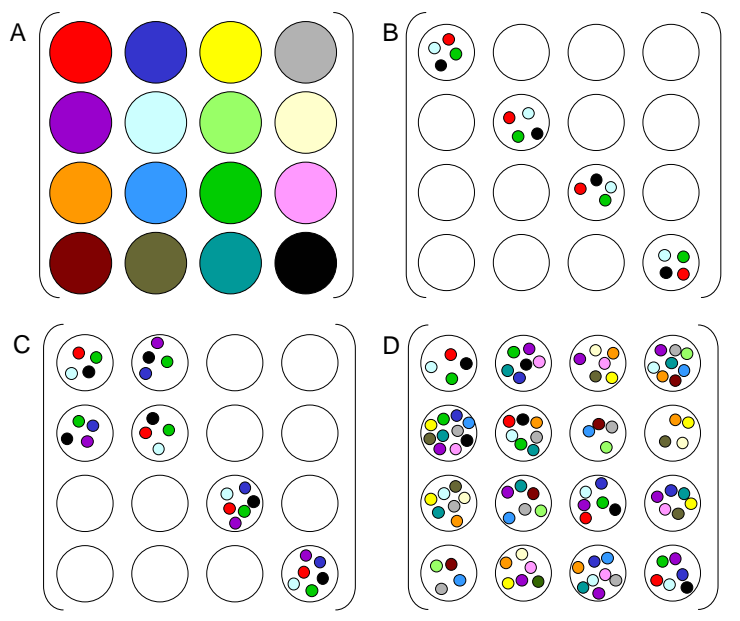

Fig.4. Part (A) defines a scheme assigning sixteen unique Q-dot barcodes to edges. Part (B) shows the readout of a simple graph. Notice that each lighted spot contains four colors, one for each edge in the graph. Part (C) shows the (superimposed) readout from two graphs. Part (D) shows the readout from an example collection of graphs defined by (3) below. 
The readout in Fig. 4(D) comes from an example collection of eight (permutation) graphs having the following adjacency matrices

$$
\begin{aligned}
& \left(\begin{array}{llll}
1 & 0 & 0 & 0 \\
0 & 1 & 0 & 0 \\
0 & 0 & 1 & 0 \\
0 & 0 & 0 & 1
\end{array}\right),\left(\begin{array}{llll}
0 & 1 & 0 & 0 \\
1 & 0 & 0 & 0 \\
0 & 0 & 1 & 0 \\
0 & 0 & 0 & 1
\end{array}\right),\left(\begin{array}{llll}
0 & 1 & 0 & 0 \\
1 & 0 & 0 & 0 \\
0 & 0 & 0 & 1 \\
0 & 0 & 1 & 0
\end{array}\right),\left(\begin{array}{llll}
0 & 0 & 1 & 0 \\
1 & 0 & 0 & 0 \\
0 & 0 & 0 & 1 \\
0 & 1 & 0 & 0
\end{array}\right), \\
& \left(\begin{array}{llll}
0 & 0 & 1 & 0 \\
0 & 0 & 0 & 1 \\
1 & 0 & 0 & 0 \\
0 & 1 & 0 & 0
\end{array}\right),\left(\begin{array}{llll}
0 & 0 & 0 & 1 \\
1 & 0 & 0 & 0 \\
0 & 1 & 0 & 0 \\
0 & 0 & 1 & 0
\end{array}\right),\left(\begin{array}{llll}
0 & 0 & 0 & 1 \\
0 & 1 & 0 & 0 \\
1 & 0 & 0 & 0 \\
0 & 0 & 1 & 0
\end{array}\right),\left(\begin{array}{llll}
0 & 0 & 0 & 1 \\
0 & 0 & 1 & 0 \\
0 & 1 & 0 & 0 \\
1 & 0 & 0 & 0
\end{array}\right) .
\end{aligned}
$$

This example collection of graphs will be used to illustrate the methods of the next section.

To end this section, we summarize the most important concept of this paper: we label the input graphs with $n^{2}$ distinct edge barcode labels, and allow the labeled inputs to hybridize on the universal chip; in the next section, optical readout of the universal biochip is obtained and then separated into $n^{2}$ partial readouts that correspond to the $n^{2}$ possible graph edges.

\section{Optical Readout from $n^{2}$ Distinct Barcode Labels}

In Subsection 4.3 we labeled a collection of DNA encoded graphs with $n^{2}$ distinct Q-bit edge barcode labels. The $n^{2}$ barcodes correspond to the complements of every possible edge in the graph.

In the laboratory, we can hybridize all labels to all inputs using an automated 384 multi-well format. We then allow the labeled collection of DNA encoded graphs to hybridize on the universal chip.

After the hybrid probes of labeled inputs are hybridized to the biochip, hybridization is detected by the fluorescence emission of Q-dots. Standard microarray protocols can be used for hybridization of biochips [13]. Traditional fluorescent microscopy can then be employed for biochip readout using a single wavelength to excite all Q-dot barcode microbeads. Images are then captured with an ImagePoint cooled CCD video camera (Photometrics), through a Labophot-2A fluorescence microscope (Nikon) [14].

\subsection{Separation of $n^{2}$ Barcode Readouts}

Next we extract from the optical readout the contributions from each one of the $n^{2}$ distinct Q-dot barcode labels. Thus, we obtain $n^{2}$ separate partial readouts of the biochip data. Our motivation is that the superposition of graphs is lessened in the partial readouts. Each partial readout corresponds to a $c_{i} \rightarrow c_{j}$ edge barcode for a particular $i$ and $j$. Such a partial readout exhibits the superposition of only those input graphs containing $c_{i} \rightarrow c_{j}$, as discussed in Subsection 4.2.

We resolve the images from each of the $n^{2}$ barcode $\mathrm{Q}$-dot beads distributed randomly over the surface of individual spots on the biochip. These images are 
about one thousand times smaller than the biochip spots. To our knowledge, no attempts have been made previously to resolve images arising from subsections of single spots on a biochip.

\subsection{Readout Constraints on Scaleup for Large Biochips}

Biochips with up to 10,000 spots can be fabricated with present technology [10]. Assuming a particular biochip has a value of $n^{2}=10,000$ (a $100 \times 100$ biochip), and each edge within the input graphs has a unique barcoded bead attached; there would be 10,000 labeled hybrid probes, each with a unique Q-dot barcode.

Scaleup is constrained by the following consideration. The diameter of a given Q-dot barcode bead can be between $0.1-5 \mu \mathrm{m}$ depending on manufacturing design, and this size is independent of the number of Q-dots contained within the bead [12]. Q-dot beads are approximately one thousand times smaller in diameter than one biochip spot (Clelland and Bancroft, personal observation). Thus, Q-dot barcode bead images could be resolved over the surface of any spot for barcode readout for rather fewer than one million barcodes. Thus, 10,000 input+label hybrid probes should be distinguishable after hybridization to a biochip.

Software that permits measurement of intensity, hue, and saturation from thousands of image objects (e.g. SigmaScan Pro, SPSS Science), can then be employed to scan each biochip spot and thus yield readout of individual barcodes.

A simple dilution of input+label hybrid probes would allow simultaneous readout of more beads. Using this approach, we can say that one laboratory step is sufficient for readout when $n^{2}=10,000$. When $n^{2}>10,000$ the number of laboratory steps is $n^{2} / 10,000$.

Our new approach for Hamiltonian path readout could replace the $O(n k)$ laboratory steps of [5] with $n^{2} / 10,000$ laboratory steps. For example, suppose $n=100$, and $n k$ has a maximum value of $n^{2}-n=9900$. In this case, 9900 laboratory steps would be replaced by one.

We know of no physical principles that would forbid scaling up universal graph readout to the level described here, and beyond.

\section{Isolating Individual DHP from $n^{2}$ Partial Readouts}

Up to this point we have presented designs and techniques for general, unrestricted graphs. We now specialize our results to permutation graphs, which are a modest generalization of directed Hamiltonian paths. A graph is said to be a permutation graph if its adjacency matrix has all 0 entries except one 1 in each row and one 1 in each column. Notice that the graphs in our example collection of graphs in (3) are all permutation graphs.

\subsection{Displaying $n^{2}$ Partial Readouts}

Consider a typical partial readout corresponding to the edge $c_{i} \rightarrow c_{j}$. Denote this $n \times n$ readout matrix by $\mathcal{R}_{i, j}$. This matrix is the superposition of all graphs 
that contain the edge $c_{i} \rightarrow c_{j}$. We can form a large array with $\mathcal{R}_{i, j}$ at the $i, j$ position. For the example collection of graphs given in (3) the partitioned matrix of partial readouts is shown in Fig. 5 .

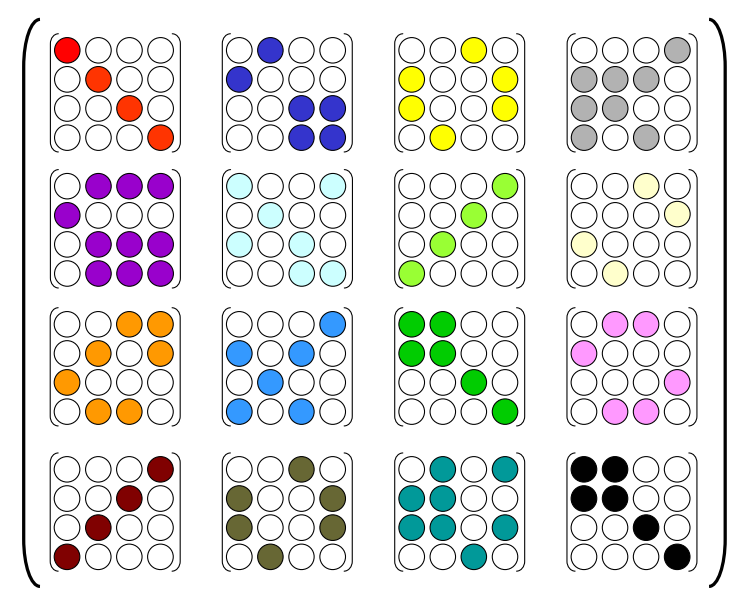

Fig. 5. For the collection of permutation graphs in (3), the $n^{2}$ partial readouts, one for each edge, are here arranged according to the color scheme in Fig. 4(A).

Since the barcode corresponding to $\mathcal{R}_{i, j}$ is placed at position $i, j$ it follows that no information is lost by replacing the information in Fig. 5 with the partitioned matrix

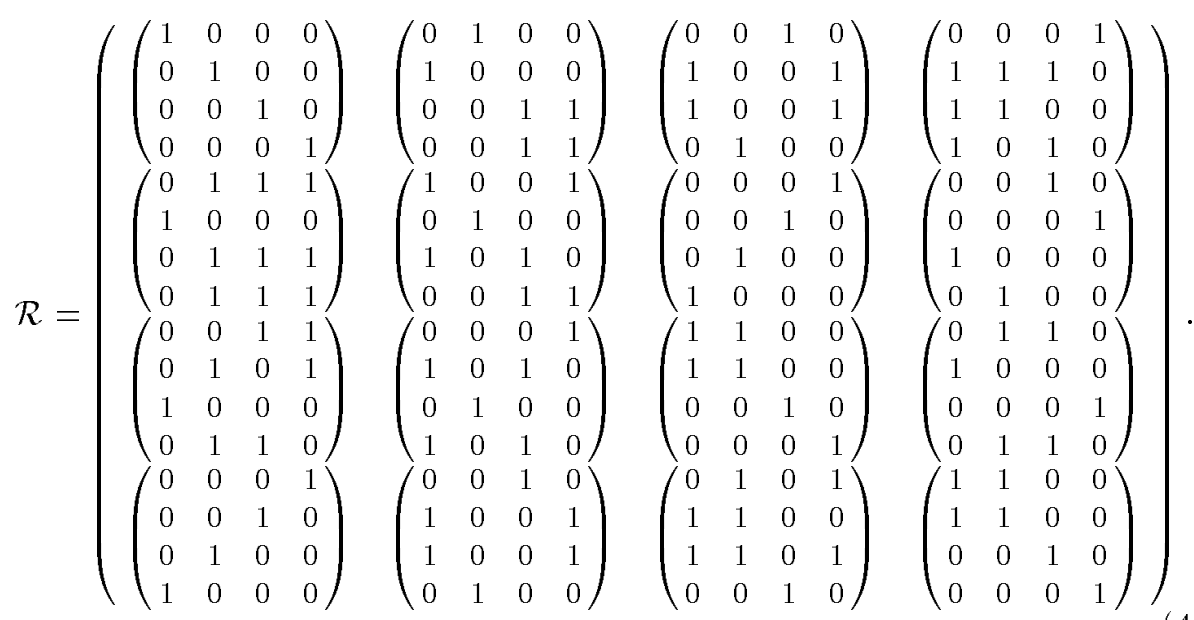

The partial readouts in (4) have their superpositions reduced compared to the same data before separation (shown in Fig. 4(D)). Indeed, some individual per- 
mutation matrices are apparent in (4):

$$
\mathcal{R}_{1,1}=\left(\begin{array}{llll}
1 & 0 & 0 & 0 \\
0 & 1 & 0 & 0 \\
0 & 0 & 1 & 0 \\
0 & 0 & 0 & 1
\end{array}\right), \mathcal{R}_{2,4}=\left(\begin{array}{llll}
0 & 0 & 1 & 0 \\
0 & 0 & 0 & 1 \\
1 & 0 & 0 & 0 \\
0 & 1 & 0 & 0
\end{array}\right), \mathcal{R}_{2,3}=\left(\begin{array}{cccc}
0 & 0 & 0 & 1 \\
0 & 0 & 1 & 0 \\
0 & 1 & 0 & 0 \\
1 & 0 & 0 & 0
\end{array}\right)
$$

These are three of the eight permutations in the input collection (3).

\subsection{Logical AND of Partial Readouts}

For any two partial readout matrices, we define $\mathcal{R}_{i, j}$ AND $\mathcal{R}_{k, l}$ to be elementwise logical AND, which is defined by 0 AND $0=0,1$ AND $0=0$ AND $1=0$, and 1 AND $1=1$. Recall that for all $i, j$, the matrix $\mathcal{R}_{i, j}$ is the superposition of all graphs that contain the edge $c_{i} \rightarrow c_{j}$. Using this fact, it can be shown that if some particular permutation matrix in a collection has a nonzero $i, j$ element and it also has a nonzero $k, l$ element, then the matrix $\mathcal{R}_{i, j}$ AND $\mathcal{R}_{k, l}$ must contain this permutation. In fact, it must contain the superposition of all permutation matrices in the collection that have both their $i, j$ and their $k, l$ elements nonzero.

Of course, the AND of two partial readout matrices has fewer (or the same) number of nonzero elements than either of the two individual matrices. That is, for any $i, j, k, l$, the matrix $\mathcal{R}_{i, j}$ AND $\mathcal{R}_{k, l}$ will often have its superposition reduced compared to $\mathcal{R}_{i, j}$ and $\mathcal{R}_{k, l}$ individually. Proceeding with the example collection of permutation graphs from (3) we AND all pairs of the 16 partial readout matrices from (4). We display the maximum number of permutations that could be contained in each of the 256 resulting matrices in the following format

\begin{tabular}{|c|c|c|c|c|c|c|c|c|c|c|c|c|c|c|c|c|}
\hline & $\mathcal{R}_{1,1}$ & & & & $R_{2}$ & & & & $\mathcal{R}_{3,1}$ & & & & $R_{4,1}$ & & & \\
\hline $\mathcal{R}_{1,1}$ & 1 & & & & & 2 & & & & & 1 & 1 & & & 1 & 1) \\
\hline $\mathcal{R}_{1,2}$ & & 2 & & & 1 & & & & 1 & & & 1 & & & 2 & \\
\hline $\mathcal{R}_{1,3}$ & & & 2 & & & & & 1 & 1 & & & & & 1 & & \\
\hline $\mathcal{R}_{1,4}$ & & & & 3 & 2 & & 1 & & & 2 & 1 & & 1 & 1 & 1 & \\
\hline $\mathcal{R}_{2,1}$ & & 1 & & 2 & 6 & & & & & 1 & 1 & 1 & & 1 & 2 & \\
\hline $\mathcal{R}_{2,2}$ & 2 & & & & & 2 & & & & & 1 & 1 & & & 1 & 1 \\
\hline $\mathcal{R}_{2,3}$ & & & & 1 & & & 1 & & & 1 & & & 1 & & & \\
\hline $\mathcal{R}_{2,4}$ & & 1 & 1 & & & & & 1 & 2 & & & & & 1 & 1 & \\
\hline $\mathcal{R}_{3,1}$ & & 1 & 1 & & & & & 2 & 2 & & & & & 1 & 1 & \\
\hline $\mathcal{R}_{3,2}$ & & & & 2 & 1 & & 1 & & & 1 & & & 1 & & 1 & \\
\hline $\mathcal{R}_{3,3}$ & 1 & & & 1 & 1 & 1 & & & & & 2 & & & 1 & & 1 \\
\hline $\mathcal{R}_{3,4}$ & 1 & 1 & & & 1 & 1 & & & & & & 2 & & & 2 & \\
\hline $\mathcal{R}_{4,1}$ & & & & 1 & & & 1 & & & 1 & & & 1 & & & \\
\hline $\mathcal{R}_{4,2}$ & & & 1 & 1 & 1 & & & 1 & 1 & & 1 & & & 2 & & \\
\hline $\mathcal{R}_{4,3}$ & 1 & 2 & & 1 & 2 & 1 & & 1 & 1 & 1 & & 2 & & & 3 & \\
\hline $\mathcal{R}_{4,4}$ & 1 & & & & & 1 & & & & & 1 & & & & & 2 \\
\hline
\end{tabular}


Collecting all the above 256 matrices $\mathcal{R}_{i, j}$ AND $\mathcal{R}_{k, l}$ that contain only one permutation, we recover exactly the eight distinct permutations that form the original collection that was given by $(3)$.

\subsection{Further Observations}

Reductions and heuristics are under development [6] for resolving superpositions of collections of permutation graphs. These are founded on three principles.

The Exclusion Principle. The partial readout $\mathcal{R}_{i, j}$ has its $i$ th row and the $j$ th column zero except at position $i, j$. Were this not the case, some permutation matrix would have more than one 1 in some row or column, which is forbidden. A consequence of the Exclusion Principle is the following.

The Verification Principle. The adjacency matrix of any permutation in a collection has non-zero entries only at positions $1, p_{1}$ and $2, p_{2}$ and $3, p_{3} \ldots$ and $2, p_{n}$, where $p_{1}, p_{2}, p_{3}, \ldots, p_{n}$ is some ordered rearrangement of $1,2,3, \ldots, n$, only if $\mathcal{R}_{1, p_{1}}$ AND $\mathcal{R}_{2, p_{2}}$ AND $\mathcal{R}_{3, p_{3}} \ldots$ AND $\mathcal{R}_{1, p_{1}}$ recreates the adjacency matrix of the permutation. We stated another principle at the beginning of the previous subsection. We repeat it here.

The AND Principle. If some permutation matrix in a collection has nonzero elements in its $i, j$ and $k, l$ positions, then the matrix $\mathcal{R}_{i, j}$ AND $\mathcal{R}_{k, l}$ contains the superposition of all permutation matrices in the collection that also have nonzero elements in both their $i, j$ and $k, l$ positions.

\section{Summary}

We have described a novel technique for reading out arbitrary graphs with up to $n$ nodes using an $n \times n$ biochip incorporating standardized DNA sequences, making this biochip universal for all graphs of this size. Multiple graphs, when simultaneously present, limit readout by superimposing their individual biochip data. We dilute superposition by scanning the distribution of $n^{2}$ different quantum dot barcode labels (one for each possible graph edge) within the spots on the universal biochip. For the special class of permutation graphs, computer-based heuristics isolated individual graphs from an example collection of graphs.

Our only laboratory process is hybridization of an unknown collection of DNA encoded graphs to quantum dot microbead barcode labels, followed by biochip hybridization and optical readout. Future scaleup to graphs with one hundred nodes seems possible using a single laboratory step because (a) pairwise ligation of one hundred standard sequences can yield ten thousand graph edge encodings, (b) printing ten thousand spots on a biochip is about the state of the art, (c) ten thousand distinct quantum dot barcode beads are on the horizon [11,12], and (d) ten thousand distinct barcode images are likely to be resolvable within each spot on a biochip. 


\section{References}

[1] L.M. Adelman. Molecular Computation of Solutions to Combinatorial Problems. Science 266:1021-1024, 1994.

[2] J. A. Rose, R. Deaton, M. Garzon, R. C. Murphy, D. R. Franceschetti, and S.E. Stevens, Jr. The effect of uniform melting temperatures on the efficiency of DNA computing. In DNA Based Computers III: DIMACS Workshop, June 23-25, 1997, pages 35-42, June 1997.

[3] M. H.. Garzon, N. Jonoska, and SA. Karl. The bounded complexity of DNA computing. Biosystems 52;63-72. 1999.

[4] CM. Lee, SW. Kim, SM. Kim and U. Sohn. DNA computing the Hamiltonian path problem. Molecular Cells. Oct 31;9(5): 464-469.

[5] DH Wood. A DNA computing algorithm for directed Hamiltonian paths. In John Koza, Wolfgang Banzhaf, Kumar Chellapilla, Kalyanmoym Deb, Marco Dorigo, DB Fogel, MH Garzon, DE Goldberg, H Iba, and R Riolo, editors, Genetic Programming 1998: Proceedings of the Third Annual Conference, July 22-25, 1998, University of Wisconsin, Madison, Wisconsin, pages 731-734, San Francisco, 1998. Morgan Kaufman.

[6] DH Wood. Determining superimposed permutation matrices from $n^{2}$ partial readouts. In preparation.

[7] R Deaton. J Chen, H Bi, M Garzon, H Rubin, DH Wood. A PCR-based protocol for in vitro selection of non-crosshybridizing oligonucleotides. These proceedings.

[8] AA BenDor, et. al., Universal DNA tag systems: A combinatorial scheme. J Comp Biol 7,503, 2000.

[9] BJ Rose, R Deaton, M Hagiya, and A Suyama. The fidelity of the tag-antitag system. Preliminary Proceedings of the 7th International Conference on DNABased Computing, 302-310, 2001. Also to appear in LNCS series, in press.

[10] E Wurmbach, T Yuen, BJ Ebersole, SC Sealfon. Gonadotropin-releasing hormone receptor-coupled gene network organization. J Biol Chem Dec 14;276(50) 47195-201, 2001

[11] E Klarreic. Biologists join the dots. Nature, 413(6855):450-452, October 2001 .

[12] M Han, X Gao, JZ Su, and S Nie. Quantum-dot-tagged microbeads for multiplexed optical coding of biomolecules. Nature Biochemistry, 19:631-635, July 2001.

[13] CL Taylor Clelland, D M P Morrow, and C Bancroft. Gene expression in prostate cancer; microarray analysis of tumor specimens and the LNCaP prostate tumor model series. American Journal of Human Genetics, 67(4):81, 2000.

[14] CL Taylor Clelland, B Levy, J M McKie, A M V Duncan, K Hirschhorn, and C Bancroft. Cloning, and characterization of human PREB: a gene that maps to a genomic region associated with trisomy $2 p$ syndrome. Mammalian Genome, 11(8):675-681, August 2000. 